\title{
SINGULARITÉS DES COURANTS D’AHLFORS
}

\author{
PAR JULIEN DUVAL \\ RÉSUMÉ. - On montre qu'une courbe algébrique chargée par un courant issu d'une courbe entière est \\ rationnelle ou elliptique. Ceci répond à une question de M. Păun. \\ (C) 2006 Elsevier Masson SAS
}

ABSTRACT. - We prove that an algebraic curve charged by a current coming from an entire curve is rational or elliptic. This answers a question by M. Păun.

(C) 2006 Elsevier Masson SAS

\section{Introduction}

Soit $X$ une variété compacte complexe hermitienne non hyperbolique au sens de Kobayashi. Elle possède alors une courbe entière, i.e. une application holomorphe non constante de $\mathbf{C}$ dans $X$.

Depuis Ahlfors (voir par exemple [1]), on sait associer à cette courbe entière un courant positif fermé. Celui-ci s'obtient comme limite de courants d'intégration normalisés $\frac{\left[\Delta_{n}\right]}{\operatorname{aire}\left(\Delta_{n}\right)}$ sur des disques concentriques de la courbe entière, choisis de sorte que la longueur de leur bord devienne négligeable devant leur aire : $\operatorname{long}\left(\partial \Delta_{n}\right)=\mathrm{o}\left(\operatorname{aire}\left(\Delta_{n}\right)\right)$.

Ces courants apparaissent notamment dans la preuve par M. McQuillan de la conjecture de Green-Griffiths pour certaines surfaces ([4], voir aussi [1]). Celle-ci stipule qu' une courbe entière dans une variété algébrique de type général dégénère algébriquement.

L'objet de cet article est de comprendre la partie singulière de ces courants. Précisément, depuis Siu (voir par exemple [2]), on sait décomposer tout courant positif fermé en une partie singulière et une partie diffuse. La partie diffuse est peu singulière : elle a des nombres de Lelong nuls hors d'un ensemble dénombrable de points. La partie singulière rend compte des courbes analytiques chargées par le courant : c'est une combinaison des courants d'intégration sur ces courbes.

À la suite de M. Păun (voir [8] qui contient aussi des réponses partielles), il est naturel de conjecturer qu'une courbe analytique chargée par un courant issu d'une courbe entière est rationnelle ou elliptique. En effet, la perte d'hyperbolicité au sens de Kobayashi due à la présence de la courbe entière doit se refléter à l'endroit où le courant se concentre le plus. Par exemple, si le courant est entièrement porté par une courbe irréductible, celle-ci est rationnelle ou elliptique par l'inégalité tautologique de McQuillan (voir [8]). Nous vérifions la conjecture dans le cas où la variété $X$ est algébrique. La méthode repose sur une propriété de relèvement des arcs de la courbe algébrique à la courbe entière. Celle-ci est liée au caractère laminaire du courant issu de la courbe entière (comparer avec [3]). 
Techniquement, il est commode d'élargir la classe des courants considérés en oubliant les courbes entières et en ne retenant que les suites de disques, voire d'unions finies de disques, dont ils sont limites :

DÉfINITION. - Un courant $T$ est dit d'Ahlfors s'il existe une suite $\left(\Delta_{n}\right)$ de réunions finies de disques holomorphes dans $X$ telle que $\operatorname{long}\left(\partial \Delta_{n}\right)=\mathrm{o}\left(\operatorname{aire}\left(\Delta_{n}\right)\right)$ et $T=\lim \frac{\left[\Delta_{n}\right]}{\operatorname{aire}\left(\Delta_{n}\right)}$.

Cette définition couvre aussi les courants issus de courbes entières après régularisation à la Nevanlinna (voir $[1,4])$. En effet un tel courant s'exprime comme limite d'intégrales de courants d'intégration sur des disques concentriques de la courbe entière. C'est donc bien un courant d'Ahlfors comme on le voit en discrétisant ces intégrales. Voici notre résultat :

THÉORÈME. - Soit $T$ un courant d'Ahlfors dans une variété algébrique $X$. On suppose que $T$ charge une courbe irréductible $C$. Alors $C$ est rationnelle ou elliptique.

La démonstration procède par l'absurde en supposant $C$ de genre au moins deux. On trouve alors un lacet $\gamma$ avec un unique point double dans $C$ - une figure huit - qui engendre dans le groupe fondamental de $C$ un groupe libre à deux générateurs. Comme $T$ se concentre sur $C$, on peut relever $\gamma$ en un graphe $\gamma_{n}$ dans $\Delta_{n}$ convergeant vers $\gamma$ et possédant beaucoup de cycles (de l'ordre de l'aire de $\Delta_{n}$ ). Comme $\Delta_{n}$ est simplement connexe, le graphe $\gamma_{n}$ va y découper beaucoup de disques (de l'ordre de l'aire de $\Delta_{n}$ ). On exhibe ainsi une suite de disques holomorphes d'aire bornée a priori dont le bord converge vers un lacet non trivial de $\gamma$. Par un résultat classique de compacité, cette suite produit un disque limite dans $C$ bordant ce lacet, c'est la contradiction.

La propriété de relèvement des arcs de $C$ à $\Delta_{n}$ s'obtient en analysant $\Delta_{n}$ sous une projection centrale via la théorie d'Ahlfors de recouvrement des surfaces (voir par exemple [6]). C'est là qu'intervient l'hypothèse d'algébricité de $X$.

Avant de préciser ceci, je tiens à remercier chaleureusement M. Păun pour m'avoir soumis ce problème. Je remercie aussi le rapporteur pour la Réf. [7]. Les auteurs y montrent par la théorie d'Ahlfors que, si une courbe entière ne s'accumule que sur une courbe algébrique, chaque composante de celle-ci est rationnelle ou elliptique.

\section{Préliminaires}

Énonçons pour commencer le peu de théorie d'Ahlfors et le résultat de compacité des disques dont nous aurons besoin. Dans la suite, les espaces projectifs seront munis de leur métrique standard.

\subsection{Un peu de théorie d'Ahlfors}

Nous renvoyons à la monographie [6] pour les détails de cette théorie. Elle a été créée par Ahlfors pour traduire en termes géométriques la théorie de Nevanlinna de distribution des valeurs.

Précisément, soit $f: \Sigma \rightarrow \Sigma_{0}$ une application holomorphe entre deux surfaces de Riemann compactes à bord. On munit $\Sigma_{0}$ d'une métrique qui induit via $f$ une pseudométrique sur $\Sigma_{0}$. Le résultat central de la théorie est une inégalité de Riemann-Hurwitz approchée pour $f$

$$
\chi^{-}(\Sigma) \leqslant s \chi\left(\Sigma_{0}\right)+c_{0} l
$$

$4^{\text {e }}$ SÉRIE - TOME $39-2006-\mathrm{N}^{\circ} 3$ 
Ici, la caractéristique d'Euler négative néglige les composantes de $\Sigma$ qui sont des disques ou des sphères, $s$ est le nombre moyen de feuillets de $f: s=\frac{\text { aire }(\Sigma)}{\text { aire }\left(\Sigma_{0}\right)}, l$ est la longueur du bord relatif de $f: l=\operatorname{long}\left(\partial \Sigma \backslash f^{-1}\left(\partial \Sigma_{0}\right)\right)$, et la constante $c_{0}$ ne dépend que de $\Sigma_{0}$ et de sa métrique.

Ahlfors en déduit son théorème des îles, l'analogue du second théorème fondamental de Nevanlinna :

THÉORÈME DES ÎLES. - Soit $f: \Delta \rightarrow P^{1}(\mathbf{C})$ une application holomorphe entre une réunion finie $\Delta$ de disques et $P^{1}(\mathbf{C})$. On se fixe $k$ disques topologiques disjoints dans $P^{1}(\mathbf{C})$ et on appelle îles de $f$ les composantes connexes de la préimage des disques sur lesquelles $f$ est propre. Alors le nombre i d'îles vérifie $i \geqslant s(k-2)-c l$.

Ici, comme plus haut, $s$ désigne le nombre de feuillets moyen de $f, l$ la longueur du bord de $\Delta$, et $c$ ne dépend que de la configuration de disques topologiques.

Il suffit en effet d'appliquer l'inégalité de Riemann-Hurwitz à $\Sigma_{0}=P^{1}(\mathbf{C})$ privé des k disques et $\Sigma=f^{-1}\left(\Sigma_{0}\right)$, sachant que le nombre de feuillets moyen de $f$ sur $\Sigma_{0}$ diffère de $s$ par un terme contrôlé par $l$.

Ceci entraîne qu'une suite de fonctions méromorphes sur des disques se comporte asymptotiquement comme un revêtement si la longueur du bord devient négligeable devant l'aire. Elle possède la propriété de relèvement suivante :

RELÈVEMENT. - Soit $f_{n}: \Delta_{n} \rightarrow P^{1}(\mathbf{C})$ une suite d'applications holomorphes entre des réunions finies $\Delta_{n}$ de disques et $P^{1}(\mathbf{C})$. On suppose, avec les notations précédentes, que $l_{n}=\mathrm{o}\left(s_{n}\right)$. On se fixe $\varepsilon>0$ et des disques topologiques disjoints dans $P^{1}(\mathbf{C})$ en nombre $k>4 / \varepsilon$. Alors un de ces disques topologiques possède au moins $s_{n}(1-\varepsilon)$ relèvements via $f_{n}$ pour $n$ assez grand.

Ici, un relèvement est une composante connexe de la préimage du disque sur laquelle $f_{n}$ est bijective.

En effet, par le théorème des îles, un des disques topologiques va posséder au moins $s_{n}\left(1-\frac{\varepsilon}{2}\right)$ îles dans sa préimage pour $n$ assez grand. Or la restriction de $f_{n}$ à chaque île est un revêtement ramifié au-dessus du disque. Ce revêtement sera donc de degré 1 pour au moins $s_{n}(1-\varepsilon)$ îles, puisque le nombre moyen de feuillets de $f_{n}$ sur ce disque est asymptotiquement $s_{n}$.

\subsection{Compacité de disques holomorphe}

Voici l'énoncé qui nous sera utile. Il remonte au moins à Gromov dans un contexte bien plus large (voir par exemple [9]).

COMPACITÉ. - Soit $f_{n}: D \rightarrow P^{2}(\mathbf{C})$ une suite d'applications holomorphes du disque unité $D$ dans $P^{2}(\mathbf{C})$. On suppose l'aire de $f_{n}(D)$ uniformément bornée. Alors, quitte à extraire,

(i) $f_{n}$ converge localement uniformément vers $f_{\infty}$ sur $D$ privé d'un nombre fini de points d'explosion;

(ii) $f_{\infty}$ se prolonge en un disque holomorphe encore noté $f_{\infty}: D \rightarrow P^{2}(\mathbf{C})$;

(iii) en un point d'explosion e, il existe une suite de disques $d_{n}$ dans $D$ tendant vers e telle que $f_{n}\left(d_{n}\right)$ converge au sens de Hausdorff vers une courbe rationnelle (une bulle).

En particulier, si l'aire de $f_{n}(D)$ reste bornée par $\frac{1}{2}$ il ne se produira pas d'explosion puisqu'une courbe rationnelle est d'aire au moins 1 . On aura dans ce cas convergence uniforme locale de $f_{n}$ vers $f_{\infty}$ sur $D$ après extraction. 


\section{Démonstration du théorème}

Notons que, par plongement de $X$ dans un espace projectif, il suffit de regarder les courants d'Ahlfors de $P^{N}(\mathbf{C})$ chargeant une courbe projective. La démonstration procède en trois étapes : la première est une réduction à $P^{2}(\mathbf{C})$, la seconde traite de la propriété de relèvement des arcs, tandis que la troisième détaille la fin de l'argument.

Pour des précisions sur les courants et leurs nombres de Lelong, nous renvoyons le lecteur à [2]. Dans la suite on ne distinguera plus les disques paramétrés de leur image géométrique. En particulier les aires des images seront comptées avec multiplicité. Que le lecteur nous pardonne cette liberté.

\subsection{Réduction de dimension}

Soit donc un courant d'Ahlfors $T$ de $P^{N}(\mathbf{C})$ chargeant une courbe projective $C$. On projette $T$ dans un hyperplan projectif par une projection $\pi$ de centre $p$. Si $p$ est choisi assez générique, le nombre de Lelong de $T$ en $p$ sera nul et la courbe $\pi(C)$ aura même genre que $C$ tant que $N \geqslant 3$. Le lemme suivant permet donc de se ramener au cas $N=2$ par projections centrales successives.

LEMME. - Le courant projeté T est un courant d'Ahlfors chargeant la courbe projective $\pi(C)$.

Démonstration. - La difficulté réside dans la singularité de $\pi$ en $p$. Précisons d'abord la définition de $\widetilde{T}$ : si $B_{r}$ désigne la boule de centre $p$ et de rayon $r$, on pose $\widetilde{T}=$ $\lim _{r \rightarrow 0} \pi_{*}\left(\left.T\right|_{\left(B_{r}\right)^{c}}\right)$. Clairement $\widetilde{T}$ charge $\pi(C)$. Reste à vérifier que c'est un courant d'Ahlfors.

Pour cela, partons de la suite de réunions finies $\Delta_{n}$ de disques holomorphes donnant $T$. Elle satisfait $\lim \frac{\left[\Delta_{n}\right]}{a_{n}}=T$ et $\operatorname{long}\left(\partial \Delta_{n}\right)=\mathrm{o}\left(a_{n}\right)$ avec $a_{n}=\operatorname{aire}\left(\Delta_{n}\right)$. La suite analogue $\widetilde{\Delta}_{n}$ pour $\widetilde{T}$ s'obtient essentiellement en projetant par $\pi$ la partie de $\Delta_{n}$ loin de $p$. Le contrôle de la longueur de son bord va provenir de l'annulation du nombre de Lelong de $T$ en $p$. Précisons ceci.

Soit $r>0$ fixé provisoirement. Dans ce qui suit, les inégalités seront à constante multiplicative indépendante de $r$ et $n$ près. Voici comment se traduit la nullité du nombre de Lelong de $T$ en $p$ pour $n$ assez grand :

$$
\operatorname{aire}\left(\Delta_{n} \cap B_{r}\right) \leqslant \varepsilon(r) r^{2} a_{n}
$$

Ici $\varepsilon(r)$ tendra vers 0 avec $r$.

L'inégalité de coaire (voir par exemple [5]) fournit alors $r_{n}$ avec $\frac{r}{2}<r_{n}<r$ et

$$
\operatorname{long}\left(\Delta_{n} \cap \partial B_{r_{n}}\right) \leqslant \varepsilon(r) r a_{n} .
$$

Remarquons que $\Delta_{n} \cap\left(B_{r_{n}}\right)^{c}$ est une réunion de disques troués. On rebouche ces trous par les composantes connexes correspondantes de $\Delta_{n} \cap B_{r_{n}}$. On obtient ainsi une réunion $\Delta_{n}^{\prime}$ de disques dont le bord consiste en deux parties, l'une incluse dans $\Delta_{n} \cap \partial B_{r_{n}}$, l'autre étant $\partial \Delta_{n} \cap\left(B_{r_{n}}\right)^{c}$. Notons $\widetilde{\Delta}_{n}$ la projection de $\Delta_{n}^{\prime}$ par $\pi$. C'est la suite recherchée.

Puisque $\pi$ dilate au plus les longueurs d'un facteur $\frac{1}{r} \operatorname{sur}\left(B_{r_{n}}\right)^{c}$, on a, d'après (2),

$$
\operatorname{long}\left(\partial \widetilde{\Delta}_{n}\right) \leqslant \varepsilon(r) a_{n}
$$

pour $n$ assez grand.

Comparons maintenant $a_{n}$ avec $\tilde{a}_{n}$ l'aire de $\widetilde{\Delta}_{n}$. Pour cela, notons $\omega$ et $\widetilde{\omega}$ les formes de Fubini-Study respectives de $P^{N}(\mathbf{C})$ et de l'hyperplan sur lequel on projette. On a $a_{n}=\int_{\Delta_{n}} \omega$ et $\tilde{a}_{n}=\int_{\Delta_{n}^{\prime}} \pi^{*} \widetilde{\omega}$. Remarquons que $\pi^{*} \widetilde{\omega}-\omega=d d^{c} u$ où $u$ est une fonction lisse hors de $p$ avec

$4^{\text {e }}$ SÉRIE - TOME $39-2006-\mathrm{N}^{\circ} 3$ 
une singularité logarithmique en $p$. En particulier $d^{c} u$ est de l'ordre de $\frac{1}{r} \operatorname{sur}\left(B_{r_{n}}\right)^{c}$. Il s'ensuit, par le théorème de Stokes et les estimées (1) et (3), que

$$
\left|\tilde{a}_{n}-a_{n}\right| \leqslant \int_{\Delta_{n} \backslash \Delta_{n}^{\prime}} \omega+\left|\int_{\partial \Delta_{n}^{\prime}} d^{c} u\right| \leqslant \operatorname{aire}\left(\Delta_{n} \cap B_{r_{n}}\right)+\operatorname{long}\left(\partial \widetilde{\Delta}_{n}\right) \leqslant \varepsilon(r) a_{n}
$$

pour $n$ assez grand. Donc long $\left(\partial \widetilde{\Delta}_{n}\right) \leqslant \varepsilon(r) \tilde{a}_{n}$.

Faisons tendre maintenant $r$ vers 0 . Après extraction diagonale sur les suites $\left(\widetilde{\Delta}_{n}\right)$ produites à $r$ fixé, on vérifie que $\widetilde{T}=\lim \frac{\left[\widetilde{\Delta}_{n}\right]}{\tilde{a}_{n}}$ et $\operatorname{long}\left(\partial \widetilde{\Delta}_{n}\right)=\mathrm{o}\left(\tilde{a}_{n}\right)$. C'est donc bien un courant d'Ahlfors.

Remarque. - Le même type d'estimée montre que l'aire de $\pi\left(\Delta_{n}^{\prime} \cap B_{r_{n}}\right)$ est contrôlée par $\varepsilon(r) a_{n}$ pour $n$ assez grand.

\subsection{Relèvement des arcs}

On s'est ramené à un courant d'Ahlfors $T$ de $P^{2}(\mathbf{C})$ chargeant une courbe projective $C$. Précisément $\left.T\right|_{C}=\nu[C]$ avec $\nu>0$.

Analysons encore $T$ sous une projection $\pi$ de centre $p$ sur une droite projective $L$. Si $p$ est choisi assez générique, on modifie comme plus haut les réunions $\Delta_{n}$ de disques définissant $T$ pour que les longueurs des bords de $\Delta_{n}$ et $\pi\left(\Delta_{n}\right)$ deviennent négligeables devant l'aire de $\Delta_{n}$, celle-ci soit équivalente à l'aire de $\pi\left(\Delta_{n}\right)$, et

$$
\lim _{r} \limsup _{n} \frac{\operatorname{aire}\left(\pi\left(\Delta_{n} \cap B_{r}\right)\right)}{\operatorname{aire}\left(\Delta_{n}\right)}=0 .
$$

Notons $s_{n}$ l'aire de $\pi\left(\Delta_{n}\right)$. C'est le nombre moyen de feuillets de $\pi: \Delta_{n} \rightarrow L$. Dans la suite on appellera $\operatorname{arc}$ un chemin injectif dans $C$ qui se projette injectivement par $\pi$ dans $L$. On dira qu'un arc $\alpha$ est $\varepsilon$-relevable si on peut l'épaissir en une bande $\beta$ dans $C$ possédant au moins $s_{n}(\nu-\varepsilon)$ relevés dans $\Delta_{n}$ après extraction, qui convergent vers $\beta$. On parlera de relevés proches. Ici un relevé de $\beta$ est une composante $\beta^{\prime}$ de $\pi^{-1}(\pi(\beta))$ dans $\Delta_{n}$ telle que $\pi: \beta^{\prime} \rightarrow \pi(\beta)$ soit bijective.

LEMME. - Tout arc $\alpha$ de $C$ peut se perturber en un arc $\varepsilon$-relevable.

Démonstration (comparer à [3]). - On se fixe un voisinage $V$ de $\alpha$ dans $C$ qui se projette injectivement par $\pi$ sur $U$. S'il est suffisamment fin, la masse de $T$ dans $\pi^{-1}(U)$ est inférieure à $\frac{\varepsilon}{4}$. Soient maintenant $k$ perturbations disjointes de $\alpha$ dans $V$, avec $k>\frac{8}{\varepsilon}$. On les épaissit un peu en $k$ fines bandes disjointes.

Appliquons la propriété de relèvement 1.1 à $\pi: \Delta_{n} \rightarrow L$ et aux $k$ disques disjoints qui sont les images par $\pi$ de ces bandes. On obtient qu'une de ces bandes $\beta_{0}$ a une projection $\pi\left(\beta_{0}\right)=\tilde{\beta}$ possédant $s_{n}\left(1-\frac{\varepsilon}{2}\right)$ relevés dans $\Delta_{n}$ pour $\pi$, si $n$ est assez grand et après extraction. Parmi ceux-ci, au moins $s_{n}(1-\varepsilon)$ sont d'aire inférieure à $1 / 2$ par le contrôle de la masse de $T$ dans $\pi^{-1}(U)$. On les note $\beta_{n, i}$. Reste à en localiser une partie près de $\beta_{0}$.

Introduisons pour cela un peu de terminologie. Soient $Q$ le cône $\pi^{-1}(\tilde{\beta})$ et $B$ l'ensemble des disques holomorphes $\beta$ de $Q$ d'aire inférieure à $1 / 2$ et se projetant bijectivement par $\pi$ sur $\tilde{\beta}$. On peut voir les disques de $B$ comme des sections de $\pi$ au-dessus de $\tilde{\beta}$.

Par 1.2 une suite dans $B$ possédera toujours une suite extraite convergente puisque la borne d'aire interdit les explosions. La limite est encore dans $B$ ou vaut $p$. Ainsi $B$ est relativement compact pour la convergence uniforme locale sur $\tilde{\beta}$, et l'espace $M$ des mesures positives sur $B$ de masse au plus 1 est compact pour la convergence faible. 
À une mesure $\lambda$ de $M$ on associe un courant "géométrique" $T_{\lambda}$ dans $Q$ par la formule $T_{\lambda}=\int_{B}[\beta] d \lambda(\beta)$. Cette application de $M$ sur l'espace $G$ des courants géométriques étant continue, on en déduit que $G$ est compact pour la topologie faible.

Revenons maintenant à nos relevés $\beta_{n, i}$. Par construction, la suite $\left(\frac{1}{s_{n}} \sum_{i}\left[\beta_{n, i}\right]\right)$ est une suite de courants géométriques $\left(T_{\lambda_{n}}\right)$ pour $\lambda_{n}=\frac{1}{s_{n}} \sum_{i} \delta_{\beta_{n, i}}$. Elle converge après extraction vers $T_{\lambda}$ dans $B$. Comme $T_{\lambda_{n}} \leqslant \frac{1}{s_{n}}\left[\Delta_{n} \cap Q\right]$ on a $T_{\lambda} \leqslant\left. T\right|_{Q}$.

Par ailleurs $\lambda$ a une masse minorée par $1-\varepsilon$ car le nombre de disques $\beta_{n, i}$ s'échappant vers $p$ est négligeable d'après (4). Donc $\pi_{*}\left(T_{\lambda}\right)>(1-\varepsilon)[\tilde{\beta}]$. Comme $\pi_{*}\left(\left.T\right|_{Q}\right)=[\tilde{\beta}]$ on en déduit

$$
\pi_{*}\left(\left.T\right|_{Q}-T_{\lambda}\right)<\varepsilon[\tilde{\beta}] .
$$

Par hypothèse $T$ charge $C$ avec un poids $\nu$ donc $\left.T\right|_{\beta_{0}}=\nu\left[\beta_{0}\right]$. Par construction $\left.T_{\lambda}\right|_{\beta_{0}}=\mu\left[\beta_{0}\right]$ où $\mu$ est la charge de $\beta_{0}$ pour $\lambda$. Donc

$$
\pi_{*}\left(\left.T\right|_{Q}-T_{\lambda}\right) \geqslant(\nu-\mu)[\tilde{\beta}] .
$$

Ainsi la charge de $\beta_{0}$ pour $\lambda$ est supérieure à $\nu-\varepsilon$. Il en est de même de la charge pour $\lambda_{n}$ de tout voisinage de $\beta_{0}$ dans $B$ pour $n$ assez grand. Autrement dit, au moins $s_{n}(\nu-\varepsilon)$ disques $\beta_{n, i}$ convergent vers $\beta_{0}$.

Un graphe $\gamma$ de $C$ est une réunion d'un nombre fini $a$ d'arcs $\alpha_{j}$ se coupant en un nombre fini $i$ de points. Deux arcs sont disjoints ou se coupent exactement une fois, soit en leurs extrémités (arcs consécutifs), soit en leurs intérieurs (arcs en croix). Le graphe $\gamma$ sera dit relevable si ses arcs sont $\varepsilon$-relevables pour $\varepsilon=\frac{\nu}{2 a+6 i}$. Grâce au lemme on peut toujours perturber un graphe en un graphe relevable en préservant sa combinatoire d'incidence. Notons $\Gamma$ la réunion des bandes $\beta_{j}$ épaississant les arcs et $\Gamma_{n}$ la réunion de leurs relevés proches dans $\Delta_{n}$. C'est l'épaississement d'un graphe $\gamma_{n}$ dans $\Delta_{n}$ qui converge vers $\gamma$.

FAIT. - On a $\chi\left(\Gamma_{n}\right) \leqslant s_{n}\left(\chi(\Gamma)+\frac{1}{2}\right) \nu$ pour $n$ assez grand.

En effet les bandes $\beta_{j}$ sont disjointes ou se coupent sur des disques qui épaississent les intersections des $\alpha_{j}$. Donc $\chi(\Gamma)=a-i$. Par ailleurs les relevés proches des bandes sont disjoints ou se recollent sur des relevés proches de ces disques. Donc $\chi\left(\Gamma_{n}\right)=a_{n}-i_{n}$ où $a_{n}$ désigne le nombre total de relevés proches de bandes et $i_{n}$ le nombre total de recollements.

Estimons ces quantités. Pour cela remarquons que les relevés proches d'une bande $\beta_{j}$ donnée (ou d'un disque) sont en nombre majoré par $s_{n}(\nu+\varepsilon)$ pour $n$ assez grand. On aurait sinon $\left.T\right|_{\beta_{j}} \geqslant(\nu+\varepsilon)\left[\beta_{j}\right]$ par passage à la limite.

Donc $a_{n} \leqslant s_{n}(\nu+\varepsilon) a$. Par ailleurs, au-dessus d'un disque intersection de deux bandes, on a au moins $s_{n}(\nu-\varepsilon)$ relevés proches provenant de chacune des bandes. Il s'ensuit nécessairement au moins $s_{n}(\nu-3 \varepsilon)$ recollements au-dessus de ce disque. Ainsi $i_{n} \geqslant s_{n}(\nu-3 \varepsilon) i$. On obtient donc par notre choix de $\varepsilon$

$$
\chi\left(\Gamma_{n}\right) \leqslant s_{n}(\chi(\Gamma) \nu+\varepsilon(a+3 i)) \leqslant s_{n}\left(\chi(\Gamma)+\frac{1}{2}\right) \nu .
$$

\subsection{Fin de l'argument}

Supposons par l'absurde $C$ de genre au moins 2. Soit $\gamma$ une figure huit (un lacet avec un unique point double) dans $C$ dont le groupe fondamental s'injecte dans celui de $C$. On reprend

$4^{\text {e }}$ SÉRIE - TOME $39-2006-\mathrm{N}^{\circ} 3$ 
ici la terminologie du paragraphe précédent. On peut toujours supposer après perturbation que $\gamma$ est un graphe relevable.

Comme $\chi(\gamma)=-1$ on a, par l'estimée précédente, $\chi\left(\gamma_{n}\right) \leqslant-\frac{s_{n} \nu}{2}$ pour $n$ assez grand. Par un argument de dualité d'Alexander, $\gamma_{n}$ découpe donc dans $\Delta_{n}$ au moins $\frac{s_{n} \nu}{2}$ disques. Or $s_{n}$ équivaut à l'aire de $\Delta_{n}$. En sélectionnant pour chaque $n$ le disque $\delta_{n}$ composante de $\Delta_{n} \backslash \gamma_{n}$ d'aire minimale, on produit ainsi une suite de disques holomorphes d'aire bornée a priori.

Analysons la convergence de $\left(\delta_{n}\right)$. Commençons par leur bord $\partial \delta_{n}$. Il est constitué d'un cycle de relevés proches d'arcs de $\gamma$. Par construction ceux-ci s'épaississent dans $\Delta_{n}$ en relevés proches des demi-bandes correspondantes dans $\Gamma$. Ces relevés forment un anneau $A_{n}$ dans $\delta_{n}$ bordant $\partial \delta_{n}$ et d'aire comparable à la longueur de $\partial \delta_{n}$. Il s'ensuit que le nombre de relevés proches d'arcs de $\gamma$ dans $\partial \delta_{n}$ est borné a priori. Donc, quitte à extraire, $\partial \delta_{n}$ converge vers un cycle non trivial d'arcs de $\gamma$. De même $A_{n}$ converge vers un anneau $A$ immergé dans $C$. En particulier le module de $A_{n}$ reste minoré. Cela permet de choisir un paramétrage holomorphe $f_{n}$ des disques $\delta_{n}$ par le disque unité $D$ tel que la préimage de $A_{n}$ contienne un anneau fixe $(r<|z|<1)$.

Appliquons l'énoncé de compacité 1.2. Comme $C$ n'est pas rationnelle, les éventuelles explosions ne peuvent se produire que dans $(|z| \leqslant r)$. Ainsi le disque limite $\delta_{\infty}=f_{\infty}(D)$ contient $A$. Par prolongement analytique il est entièrement dans $C$. Or $\partial \delta_{\infty}$ est un lacet non trivial de $\gamma$ donc non homotope à zéro dans $C$. C'est la contradiction.

\section{RÉFÉRENCES}

[1] Brunella M., Courbes entières et feuilletages holomorphes, Ens. Math. 45 (1999) 195-216.

[2] Demailly J.-P., Monge-Ampère operators, Lelong numbers and intersection theory, in: Complex Analysis and Geometry, in: Univ. Ser. Math., Plenum, New York, 1993, pp. 115-193.

[3] Dujardin R., Laminar currents and dynamics I: Structure properties of laminar currents on $P^{2}$, math.CV/0403292.

[4] MCQUILlan M., Diophantine approximations and foliations, Publ. Math. IHÉS 87 (1998) 121-174.

[5] Morgan F., Geometric Measure Theory. A Beginner's Guide, Academic Press, San Diego, 2000.

[6] Nevanlinna R., Analytic Functions, Grund. Math. Wiss., vol. 162, Springer, Berlin, 1970.

[7] Nishino T., Suzuki M., Sur les singularités essentielles et isolées des applications holomorphes à valeurs dans une surface complexe, Publ. RIMS Kyoto 16 (1980) 461-497.

[8] PAun M., Currents associated to transcendental entire curves on compact Kähler manifolds, Preprint, 2003.

[9] SIKORAV J.-C., Some properties of holomorphic curves in almost complex manifolds, in: Holomorphic Curves in Symplectic Geometry, in: Progr. Math., vol. 117, Birkhäuser, Basel, 1994, pp. 165-189. 